\title{
Performance Analysis of Block Jacobi Preconditioning Technique Based on Block Broyden Method*
}

\author{
Peng Jiang ${ }^{1}$, Geng Yang ${ }^{1}$, and Chunming Rong ${ }^{2}$ \\ ${ }^{1}$ School of Computer Science and Technology, P.O.Box 43, Nanjing University of Posts and \\ Telecommunications, 210003, Nanjing, China \\ alice20006@hotmail.com, yangg@njupt.edu.cn \\ ${ }^{2}$ Department of Electrical and Computer Engineering, \\ University of Stavanger, Norway \\ chunming.rong@uis.no
}

\begin{abstract}
The Block Jacobi preconditioning technique based on Block Broyden method is introduced to solve nonlinear equations. This paper theoretically analyzes the time complexity of this algorithm as well as the unpreconditioned one. Numerical experiments are used to show that Block Jacobi preconditioning method, compared with the unpreconditioned one, has faster solving speed and better performance under different dimensions and numbers of blocks.
\end{abstract}

\section{Introduction}

In the past few years, a number of books entirely devoted to iterative methods for nonlinear systems have appeared. The Block Broyden Algorithm was proposed and analyzed in References [1, 2]. However, the convergence speed of this algorithm is affected to some extent, for the information among the nodes is always lost. Hence, seeking for proper preconditioning methods ${ }^{[3]}$ is one of the effective ways to solve this problem. Some preconditioners have been proposed and discussed in Reference [4].

This paper introduces Block Jacobi preconditioning technique based on Block Broyden method and we name this method as BJBB. It also analyzes time complexity and applies BJBB method to nonlinear systems arising from the Bratu problem.

\section{Block Jacobi Method Based on Block Broyden Algorithm}

\subsection{General Remarks}

In the following discussion, we are concerned with the problem of solving the large system of nonlinear equations as (1):

$$
F(x)=0 \text {. }
$$

\footnotetext{
* This work was supported in part by the Natural Science Foundation of Jiangsu Province under Grant No 05KJD520144 and the Foundation of the QingLan Project (KZ0040704006).
} 
where $F(x)=\left(f_{1}, \cdots \cdot f_{n}\right)^{T}$ is a nonlinear operator from $R^{n}$ to $R^{n}$, and $x^{*} \in R^{n}$ is an exact solution. Suppose that the components of $\mathrm{x}$ and $\mathrm{F}$ are divided into $\mathrm{q}$ blocks:

$$
F=\left(\begin{array}{c}
F_{1} \\
\vdots \\
F_{q}
\end{array}\right) \quad x=\left(\begin{array}{c}
x_{1} \\
\vdots \\
x_{q}
\end{array}\right)
$$

We consider the Block Jacobi preconditioning technique based on Block Broyden method as follows:

\section{- Algorithm 2.1.1 BJBB Method}

1. Let $x^{0}$ be an initial guess of $x^{*}$, and $B^{0}$ an initial block diagonal approximation of $J\left(x^{0}\right)$. Calculate $r^{0}=F\left(x^{0}\right)$.

2. For $\mathrm{k}=0,1 \ldots$ until convergence:

2.1 Solve $B^{k} s^{k}=-r^{k}$ :

2.1.1 Calculate the Block Jacobi preconditioner M:

If the index set $S=\{1, \cdots n\}$ is partitioned as $S=U_{i} S_{i}$ with the sets $S_{i}$ mutually disjoint, then the elements $m_{i, j}$ of preconditioner $M$ is:

$$
m_{i, j}=\left\{\begin{array}{cl}
a_{i, j} & \text { if and } j \text { are in the same index subset } \\
0 & \text { otherwise }
\end{array}\right.
$$

2.1.2 Calculate the inverse of the preconditioner.

2.1.3 Transform the linear system as $\mathrm{M}^{-1} B^{\mathrm{k}} s^{k}=-\mathrm{M}^{-1} r^{k}$ and solve it by Jacobi method.

2.2 Update the solution $x^{k+1}=x^{k}+s^{k}$.

2.3 Calculate $r^{k+1}=F\left(x^{k+1}\right)$. If $r^{k+1}$ is small enough, stop.

2.4 Calculate $\left(s^{k}\right)^{T} s^{k}$ and update $B^{k+1}$ by

$$
B_{i}^{k+1}=B_{i}^{k}+\frac{r_{i}^{k+1}\left(s_{i}^{k}\right)^{T}}{\left(s^{k}\right)^{T} s^{k}} .
$$

Then set $k=k+1$, and go to step 2 .

\subsection{Time Complexity}

From Reference [2], we know that the complexity of Block Jacobi method is:

$$
U=\sum_{i=1}^{q}\left(4 n_{i}-1+2 n_{i}^{2}\right)+L(n)+R(n)
$$


here $L(n)$ means the complexity of solving q block linear equations $B_{i}^{k} s_{i}^{k}=-r_{i}^{k}$, and $R(n)$ is the calculative cost in step 2.3, Algorithm 2.1.1. From (3) we can know that the value of $\mathrm{U}$ differs in $L(n)$ for various methods.

For unpreconditioned method, we can deduce the value of $L(n)$ as follows:

$$
L_{n}(n)=k_{n} \times\left(2 \bar{n}^{3}+\bar{n}^{2}+\bar{n}\right) .
$$

where $k_{n}$ refers to the addition of number of iterations for unpreconditioned method..

For the BJBB method, we can get $\mathrm{L}(\mathrm{n})$ as follows:

$$
L_{p}(n)=q \times\left(\frac{\bar{n}^{4}}{3}+2 \bar{n}^{-3}-\frac{\bar{n}^{2}}{3}-\bar{n}\right)+k_{p} \times\left(2 \bar{n}^{-3}+\bar{n}^{2}+\bar{n}\right) .
$$

where $k_{p}$ refers to the addition of number of iteration for $\mathrm{BJBB}$ method.

\section{Numerical Experiments}

Suppose a nonlinear partial differential equation can be written as

$$
\left\{\begin{array}{l}
-\Delta u+u_{x}+\lambda e^{u}=f, \\
\left.u\right|_{\partial \Omega}=0
\end{array}(x, y) \in \Omega=[0,1] \times[0,1] .\right.
$$

It is known as the Bratu problem and has been used as a test problem by Yang in [2] and Jiang in [4]. In the following tests, we suppose $\mathrm{N}=110,150$ and 180, giving three grids, M1, M2 and M3, with 12100, 22500, 32400 unknowns, respectively. And we set block number $\mathrm{q} 1=2000, \mathrm{q} 2=800$ and $\mathrm{q} 3=2500$ for each grid. Table 1,2 , and 3 show the number of nonlinear iterations, which is denoted by " $\mathrm{k}$ " and the sum of numbers of iterations during the $\mathrm{i}$-th nonlinear iteration, which is denoted by " $\mathrm{k}[\mathrm{i}]$ ".

Table 1. Comparison of the total number of iterations in M1, q1

\begin{tabular}{ccc}
\hline & BJBB & No Preconditioner \\
\hline $\mathrm{k}$ & 3950 & 4499 \\
$\mathrm{k}[500]$ & 7106 & 13095 \\
$\mathrm{k}[1500]$ & 3533 & 7243 \\
\hline
\end{tabular}

Table 2. Comparison of the total number of iterations in M2, q2

\begin{tabular}{clc}
\hline & BJBB & No Preconditioner \\
\hline $\mathrm{k}$ & 4335 & 4713 \\
$\mathrm{k}[1700]$ & 1088 & 5601 \\
$\mathrm{k}[3290]$ & 512 & 5020 \\
\hline
\end{tabular}


Table 3. Comparison of the total number of iterations in M3, q3

\begin{tabular}{clc}
\hline & BJBB & No Preconditioner \\
\hline $\mathrm{k}$ & 4581 & 4884 \\
$\mathrm{k}[1000]$ & 4149 & 11499 \\
$\mathrm{k}[3000]$ & 1735 & 8994 \\
\hline
\end{tabular}

To judge the performance of each method, we use data shown in Table 1 as an example. During the 1500-th iteration, the following can be known:

$$
k_{p}=3533, k_{n}=7243, q_{1}=2000, \bar{n}=M_{1} / q_{1}=12100 / 2000=6
$$

According to (4), we get $L_{n}(n)=3433182$ for the unpreconditioned method. According to (5), we get $L_{p}(n)=3366642$ for BJBB method. Thus we find that $L_{p}(n)<L_{n}(n)$, so the performance of BJBB method is much better than the unpreconditioned one.

\section{Conclusions}

We have proposed Block Jacobi preconditioning technique based on Block Broyden Method for solving nonlinear systems. It shows evidently some advantages to combine Block Broyden Algorithm with preconditioners.

\section{References}

1. Yang G, Dutto L, Fortin M: Inexact block Jacobi Broyden methods for solving nonlinear systems of equations. SIAM J on Scientific Computing (1997) 1367-1392

2. Yang Geng: Analysis of parallel algorithms for solving nonlinear systems of equations. Chinese Journal of Computer (2000) 555-777 (in Chinese)

3. M. Benzi: Preconditioning Techniques for Large Linear Systems: A Survey. J Comput Phys (2002) 418-477

4. Peng Jiang, Geng Yang: Performance Analysis of Preconditioners based on Broyden Method. Applied Mathematics and Computation (Accepted for publication) 